defect. The postmortem finding of focal brain stem mineral deposits in some patients with Moebius syndrome support prenatal ischemic necrosis as an alternative explanation. (Thakkar $\mathrm{N}$ et al. Arch Neurol 1977; 34:124).

An infant with Moebius syndrome and MRI evidence of calcification in the medulla died recently at our institution and at autopsy was found to have atrophic lesions in the brain stem indicative of an ischemic pathology. (Clark HB. Personal communication). A case of a 13 year old boy with autosomal daminant congenital facial diplegia is reported fram the Division of Neurology, Ramos Mejia Hospital, University of Buenos Aires, Argentina. Thirteen members of his family were affected over four generations. Electrophysiologic studies showed blink reflex abnormalities suggesting functional damage to the brain stem. (Garcia Erro MI et al. Familial congenital facial diplegia: Electrophysiologic and genetic studies. Pediatr Neurol July/August 1989; $\underline{5}: 262-4)$.

AGENESIS OF THE CORPUS CALLOSUM

The postmortem findings in a two month old infant with the typical clinical features of Aicardi syndrome (i.e., infantile spasms, chorioretinal lacunae, and agenesis of the corpus callosum) are reported from the Division of Neurology, Saitama Children's Medical Center, Saitama, Japan. The rostrum of the corpus callosum was absent and the roof of the dilated third ventricle was covered with a thin leptomeningeal membrane. Cortical heterotropias were found adjacent to the anterior horn of the right lateral ventricle and consisted of small immature neurons. The article includes a review of five autopsied patients with this syndrome previously reported in the literature. (Hamano $S$ et al. Aicardi syndrome: Postmortem findings. Pediatr Neurol July/August $1989 ; \underline{5}: 259-61)$.

COMMENT. In these cases, a high incidence of EFG laterality and an asymetry of pathological lesions are of interest. Three of six patients had focal agenesis of the corpus callosum and three had papillama of the choroid plexus.

\title{
ARNOLD-CHIARI MALFORMATION
}

Intermittent symptams of obstructive hydrocephalus in a young woman with Chiari-I malformation are reported from the Neuro-Ophthalmology Service, Wills Eye Hospital, Philadelphia, PA. A previously healthy 26 year old waman experienced episodes of intermittent pressure headaches, dizziness, tingling in the right arm, and right anterior chest pressure for two months. These episodes lasted 5-15 minutes and occurred up to 15 times a day. They were followed by blurred vision with the appearance a "green patch" inferiorly before the left eye. Bilateral optic disc elevation was identified with indirect ophthalmoscopy. Lumbar puncture showed an opening pressure of $190 \mathrm{~mm} \mathrm{H} \mathrm{H}_{2}$. Intravenous fluorescein angiography demonstrated venous stasis, diffuse retinal hemorrhages, and disc edema interpreted as consistent with papillophlebitis. An MRI using multiple thin sagittal sections directed at the posterior fossa revealed the Chiari I malformation, and an intraventricular catheter 
confirmed that the malformation was causing an intermittent obstruction and increased intracranial pressure. In one attack in which the patient experienced several typical episodes of right hand and $r i g h t$ chest paresthesias, headache, and blurred vision the simultaneous intracranial pressure readings were elevated to a maximum of $580 \mathrm{~mm} \mathrm{H} \mathrm{H}_{2} 0$. The pressure was sustained for approximately 2 minutes; as it decreased the patient's symptams abated. Following posterior fossa decampression and a $\mathrm{Cl}$ to $\mathrm{C} 3$ laminectamy the pressure was relieved and the patient was asymptamatic. (Vrabec TR et al. Intermittent obstructive hydrocephalus in the Arnold-Chiari malformation. (Ann Neurol September 1989; 26:401-404).

OOMENT. Al though this patient is outside the pediatric age group, Chiari I malformation becomes symptomatic in children and the transient nature of the signs and symptams may prove misleading in diagnosis. Conventional CT and MRI may fail to reveal the malformation and posterior fossa directed MRI using multiple thin sagittal sections including the midline view may be necessary for diagnosis.

\section{ORIGINS OF CEREBRAL PALSY}

The causes of cerebral palsy were determined in a prospective study of 43,437 full term children from data collected in the Collaborative Perinatal Study of the National Institute of Neurological and Communicative Disorders and Stroke, Bethesda, MD, and published from the Department of Pathology and Center for Biostatistics and Epidemiology, M.S. Hershey Medical Center, Pennsylvania State University College of Medicine, Hershey, PA. The diagnosis of cerebral palsy had been made by neurologic examination at seven years of age in 127 and at one year of age in 23 who then died before the seven year follow-up. The incidence of cerebral palsy in the group studied was 1 in 290 $(0.34 \%)$. Based on attributable risk estimates, congenital malformations explained over half (53\%) of the cases of quadriplegic cerebral palsy. Only $14 \%$ of the cases of quadriplegic CP was attributable to birth asphyxia and $8 \%$ to CNS infections. Of 116 nonquadriplegic patients with cerebral palsy congenital disorders explained about one-third and CNS infections about 1 in 20 ; no cause could be identified for nearly $60 \%$ and birth asphyxia was not a significant antecedent. Characteristic consequences of birth asphyxia were more often the result of nonasphyxial disorders. These included meconium in the amniotic fluid, low 10 minute Apgar scores, neonatal apnea spells, seizures, persisting neurologic abnormalities, and slow head growth after birth. Congenital disorders explained nearly four times as many cases of quadriplegic CP then did birth asphyxia (attributable risks, $53 \%$ vs $14 \%$, respectively). Congenital disorders were the only factor that explained a large number of the nonquadriplegic CP cases. Oxytocin, whose use was followed by an increased frequency of neonatal seizures, was not followed by a corresponding increase in the frequency of CP. (Naeye, RL et al. Origins of cerebral palsy. AJDC October 1989; 143:1154-1161).

COMMENT. Many previous studies have invoked asphyxia as the cause of CP on the presence of fetal distress and low Apgar scores. 